telephony, but Zwaardemaker, not content with subjective listening at the thermotelephone, secured objective measurements by employing the Rayleigh principle of the acoustic mirror.

In conclusion, the records of the Congress point to activity along lines already initiated, and there was little evidence of the breaking of new ground Thus the researches of the 'configurationists, useful as they are, as a contrast to the work of some ' atomists,' could yet be assimilated by many outside their camp without sacrificing any psychological principles.
LL. W. J.

\title{
Coal Blending.
}

THE gradual exhaustion of the more easily mined seams of coal in Great Britain, and other factors which are resulting in increasing costs of mining and transport, are combining to focus attention on the necessity of employing more scientific methods in the preparation and utilisation of coal. In coal carbonisation, the main object of the coke oven is to produce coke possessing the special properties required by the metallurgical industries; whereas the chief aim of the gas industry has been to manufacture gas of the quality required for domestic and industrial purposes, and coke as a secondary product has not received the attention it deserves. The possibility of producing coke with the qualities desirable in a solid smokeless fuel for domestic use is now being realised, and coke quality and structure are consequently being given much more consideration.

Many attempts have been made to manufacture a solid smokeless fuel, containing a comparatively high percentage of volatile matter, by the carbonisation of coal at low temperatures ; and satisfactory products have been obtained, but the costs of the processes have been so great that it has not yet been possible to establish them on a sound commercial basis.

In high temperature carbonisation processes, largely on account of the low thermal conductivity of coal, the charge adjacent to the retort walls is fully carbonised before any marked decomposition of the coal in the centre of the charge has commenced. The exposure of the coke to high temperatures for long periods, and the small amount of volatile matter, both operate in the direction of rendering the product difficult to ignite. The work of numerous investigators, however, has led definitely to the conclusion that these are not the only factors which influence the quality of coke as regards relative ignitability, combustibility, and reactivity. Other factors of importance include the character of the coal carbonised, the state of division of the coal, the method, speed, and duration of heating and the temperature of carbonisation.

The character of the coal to be carbonised may be controlled by grinding and mixing coals of different coking properties with one another or with coke breeze or other substances, in predetermined proportions. The main effects of efficient blending are more rapid heat penetration of the charge and the production of a harder and more easily combustible coke. The method, speed, and duration of heating are largely determined by the design of carbonisation plant.
In a recent paper to the Iron and Steel Institute, ${ }^{1} \mathrm{Mr}$. David Brownlie presents a useful survey of the subject of coal blending; that is, the mixing of coals with one another and with other carbonaceous materials, such as low temperature fuels, coke, pitch, etc., especially in connexion with carbonisation. A summary of the paper is not attempted in this article, since the paper, although extending to forty-two pages, is in itself a summary, and the titles of the sections alone occupy more than one page.

Some coking coals are so constituted that they do not require blending for the production of good high temperature coke, but the amount of coal of this type is limited. Scientific blending would render many more coals available for use in the carbonisation industries. It must be emphasised, however, that the blending of coals on a commercial scale requires close attention, as the correct blend for a particular process and temperature of carbonisation would not necessarily be suitable for other processes or temperatures. Other factors, in addition to the amounts of resinous material in the coals constituting a blend, must also be taken into account. For example, the investigations of the Joint Research Committee of the University of Leeds and the Institution of Gas Engineers have shown that the degree of fineness of the coal carbonised exerts important influences. Further, the work of C. B. Marson and J. W. Cobb has demonstrated that the character of the coke may be influenced considerably by the nature of the ash constituents. Iron oxide, calcium oxide, and sodium carbonate have marked beneficial effects, but certain other substances examined were apparently inert.

The application, on a commercial scale, of the results obtained in laboratory investigations of coal blending presents certain difficulties. In addition to the installation of grinding and mixing machinery, increased bunker capacity would be required. It would also be necessary to ensure that only small variations in the quality of coal from a particular colliery occurred in different consignments. The advantages obtained by blending must also compensate for the additional cost of the preparation of the blends ; and in this connexion it must be pointed out that the depreciation of grinding machinery, when coke is one of the constituents of the blend, may be considerable.

A. PARKER.

1 Coal Blending. A General Review of Principles as Applied both to High and Low-Temperature Carhonisation, by D. Brownlie. Iron and Stee Institute, June 1926 .

\section{Physical Phenomena and Molecular Orientation at Interfaces.}

THE behaviour and properties of interfaces were discussed at a conference arranged by the Faraday Society on October 1 . The subject is of fundamental importance in the science of colloids, and it is only by a better appreciation of the former that we can hope to unravel the peculiarities of such complex systems as are to be found in natural colloidal materials. The liquid-gas and liquid-liquid interfaces are more simple than those containing a solid surface, since liquids present equipotential surfaces. There is little doubt that the concept of a unimolecular layer of orientated molecules as constituting the surface layer of an insoluble oil on the surface of water is correct, and many investigations are being made on the conditions of formation and stability of these orientated, two-dimensional systems in their solid, liquid, vapour, and gaseous states of aggregation. All the usual three-dimensional phase phenomena, such as allotropy or the process of vaporisation, have been shown to have their two-dimensional prototypes. For soluble substances the surface composition can only be calculated by means of the Gibbs' equation, a

NO. 2973, VOL. I I 8] 